\title{
Advertisement Evaluation Based On Visual Attention Mechanism
}

\author{
Yu Xiao ${ }^{1,2}$, Peng Gan ${ }^{1,2}$, Yuling Sun ${ }^{1,2}$ \\ ${ }^{1}$ School of Electronics and Information Engineering, Tianjin Polytechnic University, Tianjin 300387, \\ @China; \\ ${ }^{2}$ Tianjin Key Laboratory of Optoelectronic Detection Technology and System, Tianjin 300387, \\ China. \\ a845297648@qq.com
}

Keywords: Advertisement evaluation, visual attention, saliency detection, fixations.

\begin{abstract}
Evaluation methods of advertisement design are important in Advertising, This paper proposed a novel approach to advertisement evaluation based on visual attention. Firstly saliency detection algorithm is used to detect the salient region of advertisement, then an advertisement is determined as attractive if the detected salient regions are overlapped with the interested regions of the advertisement. Based on the salient regions, the fixations are generated in sequence combined with the competition mechanism of WTA and inhibition of return mechanism. The fixations reflects the intensity of each salient region in the advertisement, which determines the priority of the visual effect on each visual element. The experimental result shows that this evaluation method can basically simulate the attention mechanism of human visual system to evaluate the advertisement objectively.
\end{abstract}

\section{Introduction}

With the development of society, the consumer psychology of consumers is becoming more and more mature, the channel of information acquisition is becoming more and more diversified. Rational consumption is more prevalent than impulsive consumption. Existing advertisements are not only used to guide consumption, but also to shape and beautify their products and promote their corporate image. From the perspective of marketing management, advertisements have become to be an important method to promote the brand image [1]. So the study on the effect of advertisement design is the trend of advertising marketing at present, it can strengthen the scientific and rationality of advertising marketing and make the design of advertisements more well-directed, so that it would achieve the best effect of advertisement in actual application.

The evaluation of an advertisement about its attractiveness is one of the most fundamental and important tasks in the field of advertising psychology. Previously works in this field focus mainly on the activity of the customer's eyes captured by eye tracking devices [1, 2, 3]. Wedel and Pieters [1] divided an advertisement into three elements (brand, pictorial and text), and recorded the frequency of eye fixations falling into each element of the advertisement. They found that viewers are more likely to fixate on the pictorial element, followed by the text and the brand element. They also found that the memory of advertised brand is related to the frequency of eye fixations on the advertisement.

The eye tracking device records the actual activity of the viewer's eyes in real time. However, such expensive devices are not available in many cases, and it is also annoying to wear the device in most situations. Therefore some researchers concentrate on the visual saliency or attention of the 
advertisement stimuli to overcome the problem of using the eye tracking device in advertisement evaluation $[4,5,6,7]$.

In this paper, a novel advertisement evaluation based on visual attention mechanism is proposed, which provides a guarantee for the objectivity of the evaluation method. The experimental results show that this method can effectively display the visual stimuli of the interested regions in the advertisement, so that it can provide the data feedback for the advertisement design, which can be used as an assistant method in the advertisement evaluation.

\section{An advertisement evaluation method based on visual attention}

The visual attention mechanism is the key in human visual system. To process the important information faster and more efficient, the eyes always selectively focuses on the most attractive regions in the scene [13]. In order to integrate the visual attention mechanism into the practical application, the domestic and foreign scholars put forward the saliency detection algorithm, the detected saliency regions and fixations simulate the visual attention mechanism. According to the theory of psychometric measurement, it shows that most of the viewer's fixations are focused on the interested regions, the saliency detection algorithm based on the visual attention mechanism simulate the selective of the human visual system to detect the interested regions of human visual system [8]. Therefore, in this paper, the saliency detection algorithm and the theory of visual psychological measurement are combined to evaluate the advertisement objectively.

Saliency detection model. Itti et al. proposed a visual computation model based on spatial domain with the local feature contrast. The color, intensity and orientation of the input image are expressed in different scales. The feature saliency maps are obtained by multi-scale visual features calculated by the center-periphery operator, and finally they are combined into a saliency map [13]. This model based on the biological structure of the calculation method is more suitable for simulation of human visual system [14].

Feature extraction. The features of the color, intensity and orientation of the input image are decomposed by the multi-channel multi-scale filter sets. Then the feature maps is extracted from the different feature information by using the central peripheral difference method. Because the regions attracting the visual attention is the most "distinctive" part, which is more prominent than the rest of the surrounding part. It convert the difference of the feature map at the coarse scale to the feature map at the fine scale, and then perform the point-to-point subtraction. The fine scale can find the high frequency part, the coarse scale can find the low frequency part, the fine scale feature chart represents the central area, and the coarse-scale feature map represents the surrounding area.

Now we define $\Theta$ as the central peripheral difference operator. In this model, the pixels belonging to the scale 2, 3, $4(c \in\{2,3,4\})$ are called the central part, and the pixels belonging to the scale $s$ $=c+\delta, \delta \in\{3,4\}$ are called peripheral parts.

For the intensity feature image, six intensity features are calculated from the central difference:

$$
I(c, s)=|I(c) \Theta I(s)|
$$

For the color feature image, we define four color channels: $R=r-(g+b) / 2, G=g-(r+b) / 2$, $B=b-(r+g) / 2, Y=(r+g) / 2-(r-g) / 2-b, R(\sigma), G(\sigma), B(\sigma)$ and $Y(\sigma)$ Gaussian pyramids are generated. The color feature maps $R G(c, s), B Y(c, s)$ is defined as:

$$
\begin{aligned}
& R G(c, s)=|(R(c)-G(c)) \Theta(G(s)-R(s))| \\
& R Y(c, s)=|(B(c)-Y(c)) \Theta(Y(s)-B(s))|
\end{aligned}
$$


Then twelve color feature maps are calculated.

For the orientation feature image, firstly the Gabor filter is used to extract the orientation feature, and the orientation Gabor pyramid $O(\sigma, \theta), \theta \in\{0, \pi / 4, \pi / 2,3 \pi / 4\}$ is calculated with the intensity image $I(\sigma) . \theta$ are the four default orientations, each orientation represents the six feature maps, and the orientation feature maps $O(c, s, \theta)$ is defined as:

$$
O(c, s, \theta)=|O(c, \theta) \Theta I(s, \theta)|
$$

In summary, 42 feature maps are achieved by feature extraction, including six intensity feature maps, 12 color feature maps and 24 orientation feature maps.

Generating salient maps with feature fusion. Feature saliency maps at different scales are achieved by feature extraction at different scales, now the feature saliency maps in the same feature under different scales are merged into a feature map according to a certain merging strategy. In this paper, the local feature iteration method that convolve the Gaussian difference function and the normalized eigenvalues is used to compute the final feature saliency map, and is obtained. From the biological point of view, the Gaussian difference function are similar to the organization of the human visual cortex which shows it has the biological rationality, and is suitable for simulating the human visual system. After the feature fusion strategy, three feature saliency maps are obtained, as shown in Fig.1, which are the intensity feature saliency map, the color feature saliency map and the orientation feature saliency map respectively. The final saliency map was obtained by combining the three feature saliency maps.

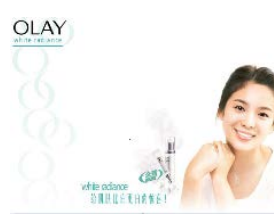

(a)

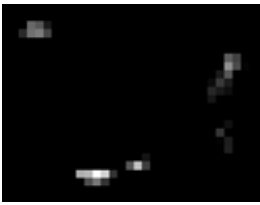

(b)

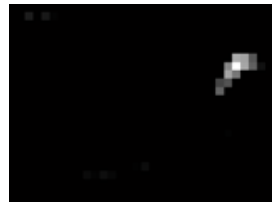

(c)

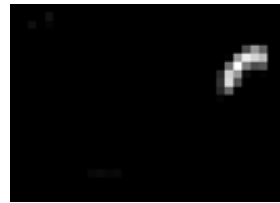

(d)

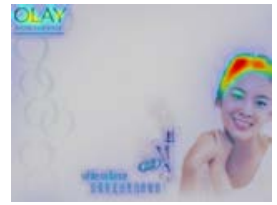

(e)

Fig.1 Plots of the salient images of the plane advertisement images. (a) the original image; (b) a color feature saliency map; (c) intensity feature saliency map; (d) orientation feature saliency map;

(e) saliency map

The generating fixations and its transfer. The pyramid model is constructed based on non-uniform sampling and multi-scale analysis. The Winner detected in the WTA (winner take-all) competition mechanism is the most noticeable fixation by using the coarse-to-fine detection strategy, which is the fixation with the highest degree of pixel.

Since the most noticeable fixation in the WTA mechanism will always be the Winner, then the fixation will not be transferred. Combining with the inhibition of return mechanism, once the Winner is generated, saliency maps will receive an input that its spatial distribution is similar to the differential Gaussian function. The suppression center is located at the Winner, and the neighborhood centered on the Winner will be masked and then the fixation can transfer to the next one. As shown in Fig.2, with the transfer of fixations, the corresponding areas of fixation in the saliency map are suppressed, and then the WTA mechanism generates the next fixation in the suppressed saliency map. In this paper, the flow diagram of the saliency detection is shown in Fig.3. 


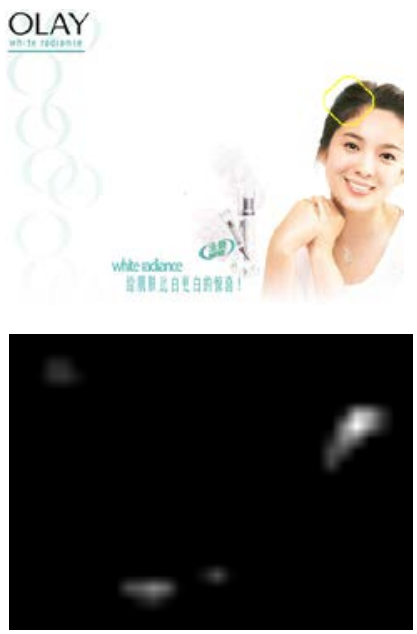

(a) The fixation I
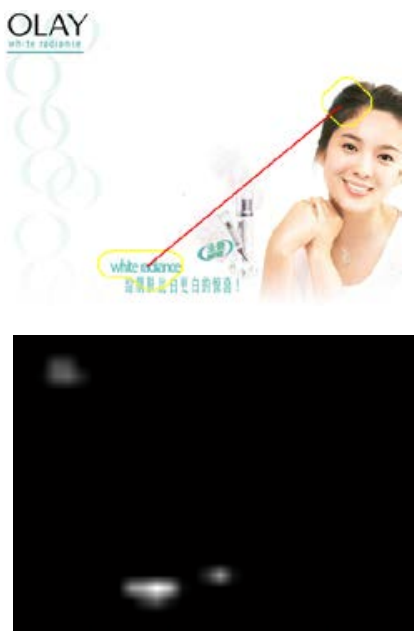

(b) The fixation II
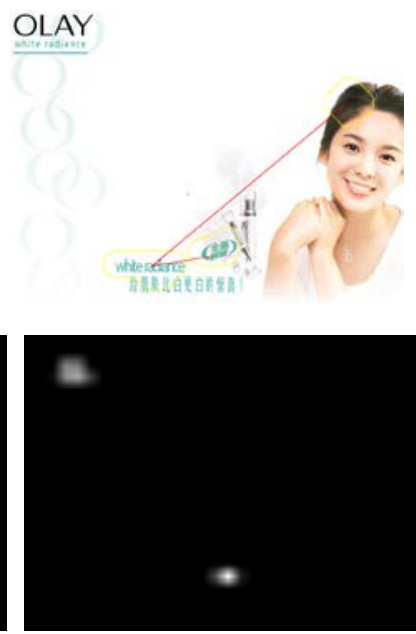

(c) The fixation III

Fig.2 Example: the transfer process of fixations

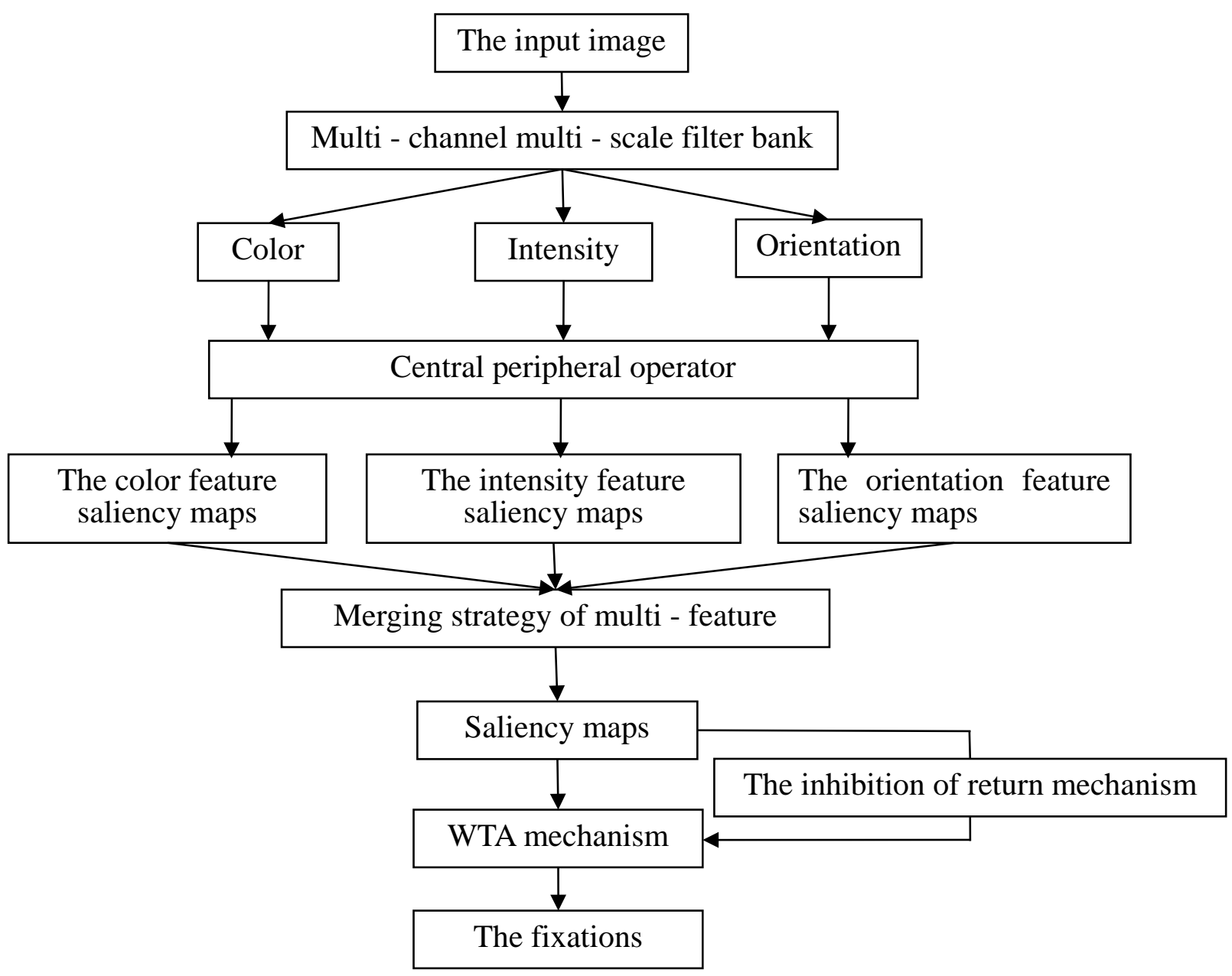

Fig.3 Flow diagram of saliency detection

Advertisement evaluation. In the visual perception process, the eyes firstly focus on a fixation of the image, and then the visual system further analyzes and processes the information in the vicinity of the fixation to achieve information about the salient region [8]. Therefore, an 
advertisement is determined as attractive if the detected salient regions are overlapped with the interested regions of the advertisement. According to the theory of visual psychology, the consumers can achieve a lot of useful information during the fixation, and the first fixation position of the consumers often affects the advertisement effect [9,10,11].

According to the experimental results of the algorithm of saliency detection, the fixations hit the interest regions of advertisements are listed. the designed interest regions are determined as attractive with the order and the number of fixations in the interest regions [12].

\section{Experimental results}

240 images are collected by searching the Google with keyword "classic advertisement" and "failure advertisement" in both English and Chinese, and the search result are carefully examined to avoid potential duplication. Four students annotate the product and its trademark in each advertisement as salient regions. The effectiveness of an advertisement is measured by examining the frequency of fixations falling in these salient regions.

Comparisons of saliency maps. The saliency regions obtained by our method are compared with the eye movement data, as shown in Fig.4, the saliency regions obtained by this method are close to the eye tracker data. It shows that our method can detect the interest regions of advertisements. In the initial advertisement evaluation, it can visually display the interest areas of advertisements.

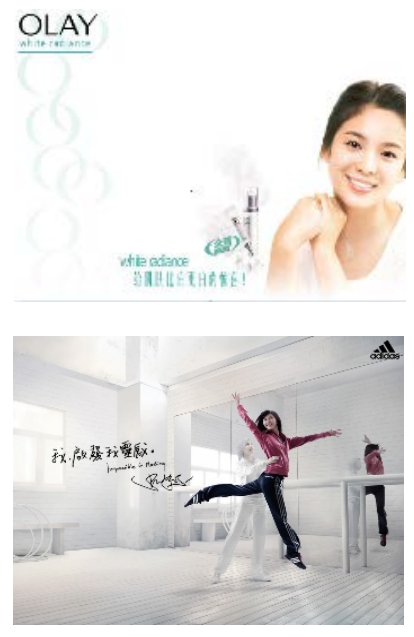

(a)
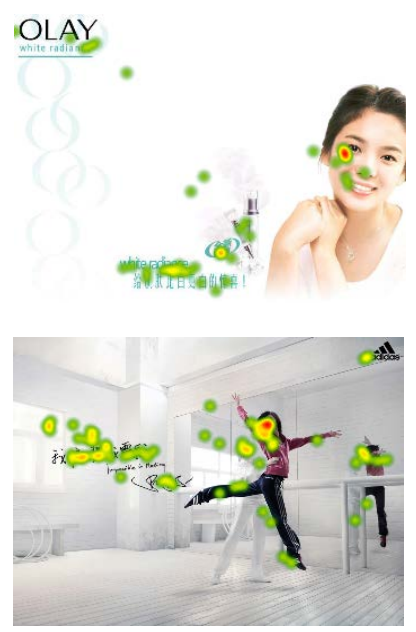

(b)
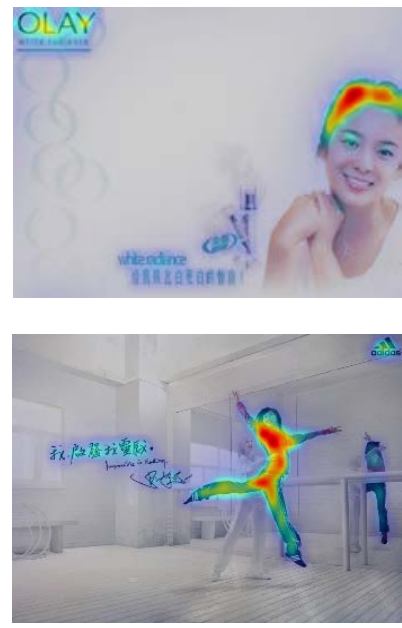

(c)

Fig.4 Comparisons of saliency maps. (a) The original image; (b) Eye tracker experimental data; (c) The results of our method.

The order of fixations in the interest regions. As shown in Fig.5, the order of fixations for the first time falling into the interest regions are listed. Table 1 summarizes the average order of the method and the eye tracker's results for the first time in the interest regions. The data show that this method is more accurate and effective than the eye tracker in terms of the fixation order area. 
(a)

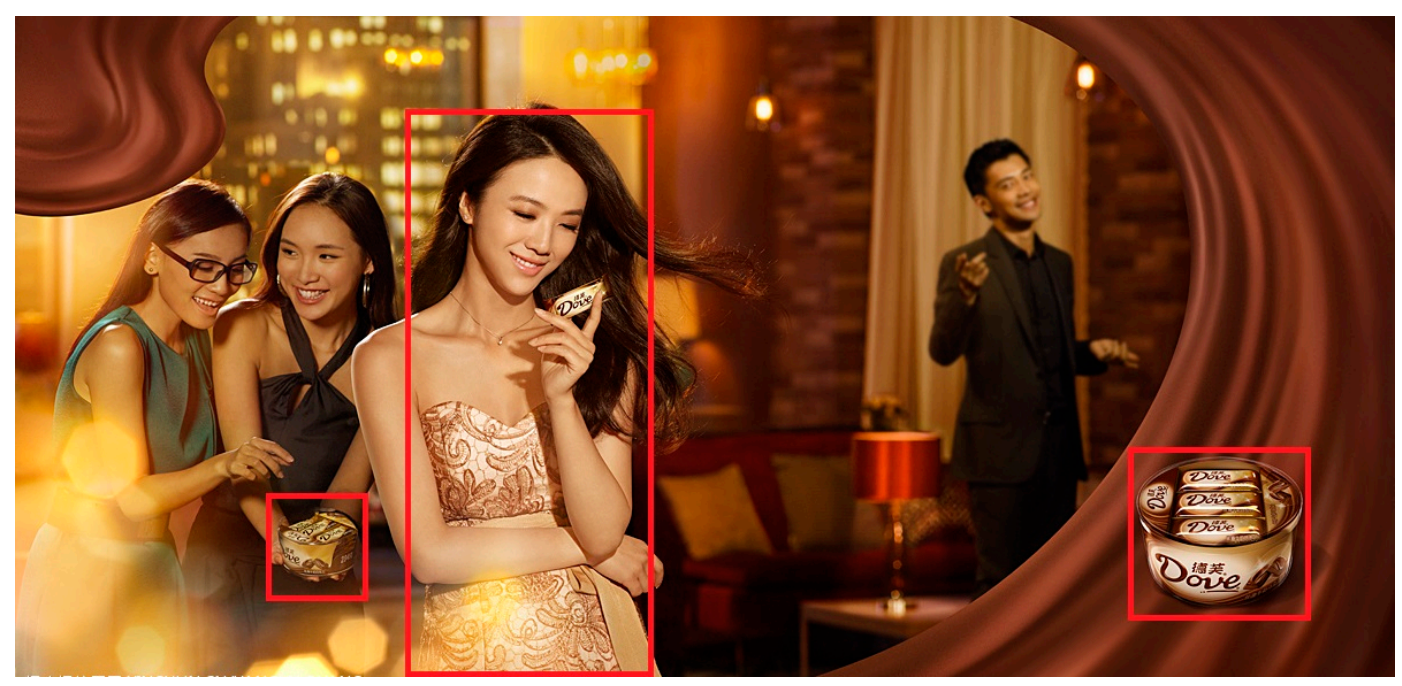

(b)

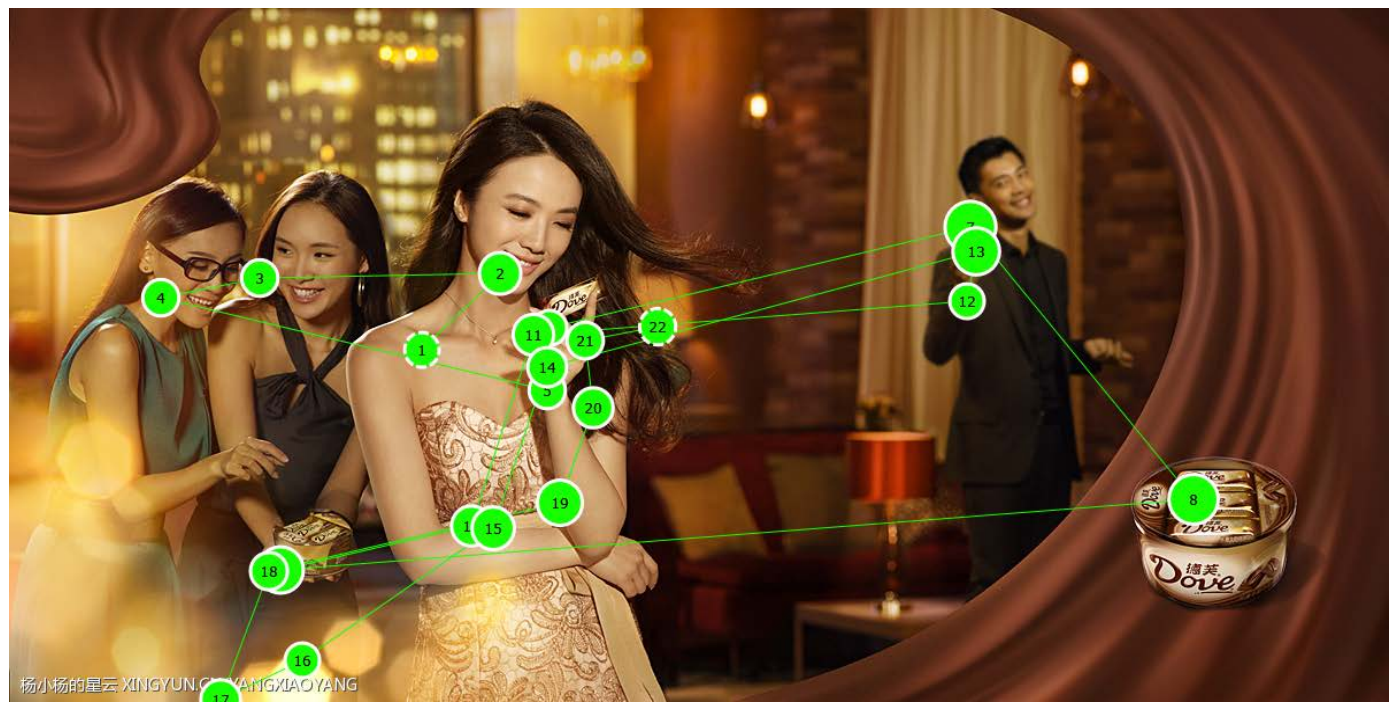

(c)

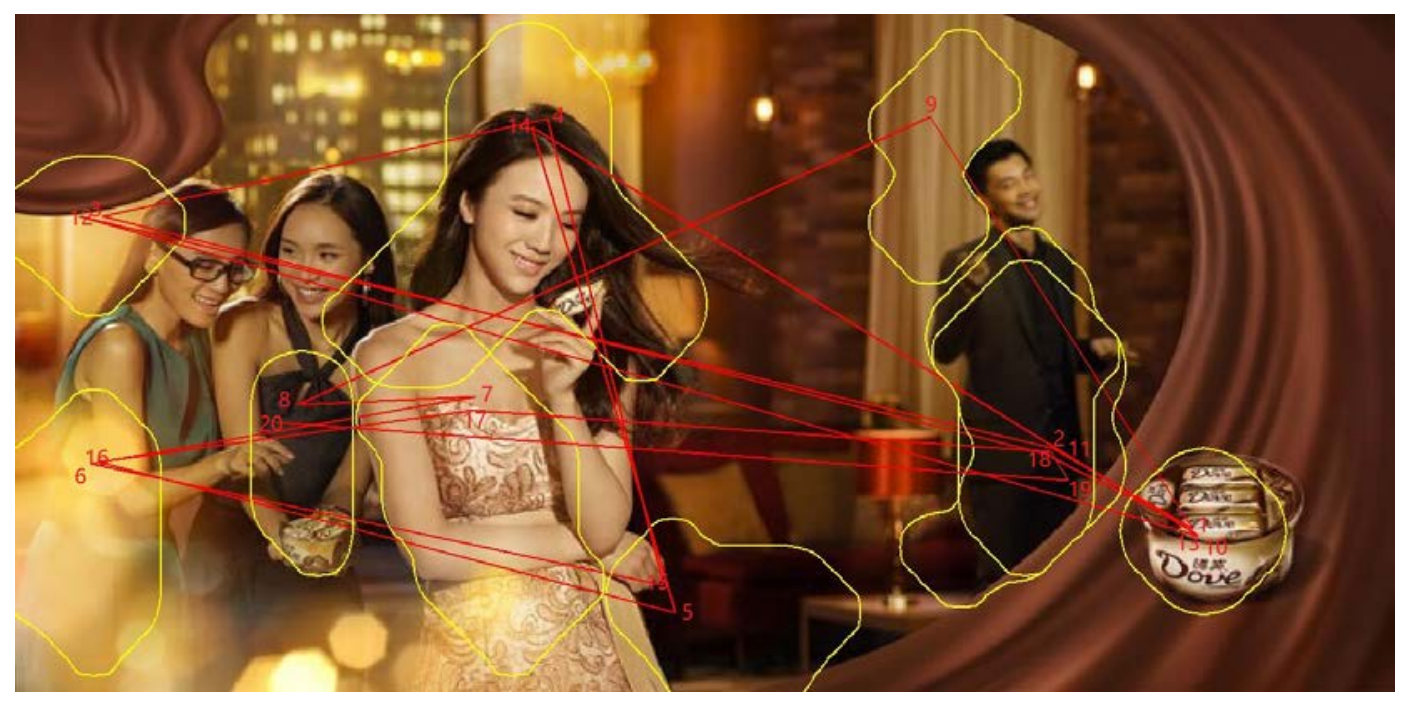

Fig.5 the comparison of the fixations order. (a) Labeled maps; (b) eye tracking data; (c) the results of this method. 
Table 1 Comparison of the average fixation order

\begin{tabular}{c|c|c|c|c}
\hline & \multicolumn{2}{|c|}{ Classic advertisements } & \multicolumn{2}{c}{ Failure advertisements } \\
\hline & Paper method & Eye tracker data & Paper method & Eye tracker data \\
\hline Labeler 1 & 1.22 & 1.62 & 4.65 & 5.58 \\
\hline Labeler 2 & 1.22 & 1.58 & 4.65 & 5.26 \\
\hline Labeler 3 & 1.22 & 1.67 & 4.65 & 5.24 \\
\hline Labeler 4 & 1.22 & 1.56 & 4.65 & 5.35 \\
\hline
\end{tabular}

The number of fixations in the region of interest. As shown in Fig.5, the number of fixations in the interest regions is counted. Because the number of fixations in the eye tracker is about twenty times, then twenty fixations are generated in our method. Table 2 shows the average number of our method and eye tracker fall into the interest regions. The data show that the performance of our method is close to that of the human beings, and our method can detect the salient regions more efficiently.

Table 2 Comparison of average fixation times

\begin{tabular}{c|c|c|c|c}
\hline & \multicolumn{2}{|c|}{ Classic advertisements } & \multicolumn{2}{c}{ Failure advertisements } \\
\hline & Paper method & Eye tracker data & Paper method & Eye tracker data \\
\hline Labeler 1 & 10.83 & 10.12 & 6.58 & 5.83 \\
\hline Labeler 2 & 10.83 & 10.24 & 6.58 & 6.25 \\
\hline Labeler 3 & 10.83 & 9.86 & 6.58 & 5.47 \\
\hline Labeler 4 & 10.83 & 10.56 & 6.58 & 6.51 \\
\hline
\end{tabular}

\section{Conclusion}

This paper proposes an advertisement evaluation method based on visual attention mechanism. The proposed method achieves satisfying results on the task of advertisement evaluation by taking advantage of the visual attention. Future works include integration of other high level features.

\section{References}

[1] M. Wedel and R. Pieters, "Eye fixations on advertisements and memory for brands: A model and findings,” Marketing Science, vol. 19, no. 4, pp. 297-312, 2000.

[2] K. Rayner, C.M. Rotello, A.J. Stewart, J. Keir, and S.A. Duffy, "Integrating text and pictorial information: eye movements when looking at print advertisements,” JExp Psychol Appl, vol. 7, no. 3, pp. 216-226, Sep 2001.

[3] J.E. Russo and F. Leclerc, "An Eye-Fixation Analysis of Choice Processes for Consumer Nondurables,” Journal of Consumer Research, vol. 21, no. 2, pp. 274, 1994.

[4] G. Guido, The Salience of Marketing Stimuli: An Incongruity-salience Hypothesis on Consumer Awareness, Springer, 2001.

[5] C. Pechmann and D.W. Stewart, "The Effects of Comparative Advertising on Attention, Memory, and Purchase Intentions,” Journal of Consumer Research, vol. 17, no. 2, pp. 180, 1990.

[6] O. Loginova, "Competing for consumers attention: Advertising when consumers have imperfect memory,” University of Missouri-Columbia Mimeograph, March,2005.

[7] M. Stegeman, Advertising in Competitive Markets, Department of Economics, University of 
North Carolina, 1988.

[8] N. Hu. Based on the visual attention mechanism of the apparel print ads[D]. Donghua University, 2010.

[9] X.Y. Zhao. Advertising principles and practice. Northeast University of Finance and Economics Press .2006: 48-49

[10]B. Jiang, Z.H. Zeng. Evaluation of advertising effectiveness, the first edition. Beijing: China Radio and Television Press, 2002: 54-55.

[11]H. Wang. Comprehensive evaluation model of Internet advertising effectiveness. Journal of Wuhan Commercial Service College, 2012,04: 49-54.

[12]J. He. Advertising and visual communication. Beijing: China Light Industry Press, 2001: 52-53.

[13]Itti L, Koch C. Computational modeling of visual attention. Nature Reviews Neuroscience, 2001, 2(3): 194-203.

[14]Simone Frintrop, Thomas Werner. Traditional Saliency Reloaded: A Good Old Model in New Shape. In CVPR, 2015. 